\title{
Estimation of the Readily Releasable Synaptic Vesicle Pool at the Drosophila Larval Neuromuscular Junction \\ Pragya Goel ${ }^{\#}$, Xiling Li* and Dion Dickman*
}

\author{
Department of Neurobiology, University of Southern California, Los Angeles, USA \\ *For correspondence: dickman@usc.edu \\ \#Contributed equally to this work
}

\begin{abstract}
[Abstract] Presynaptic boutons at nerve terminals are densely packed with synaptic vesicles, specialized organelles for rapid and regulated neurotransmitter secretion. Upon depolarization of the nerve terminal, synaptic vesicles fuse at specializations called active zones that are localized at discrete compartments in the plasma membrane to initiate synaptic transmission. A small proportion of synaptic vesicles are docked and primed for immediate fusion upon synaptic stimulation, which together comprise the readily releasable pool. The size of the readily releasable pool is an important property of synapses, which influences release probability and can dynamically change during various forms of plasticity. Here we describe a detailed protocol for estimating the readily releasable pool at a model glutamatergic synapse, the Drosophila neuromuscular junction. This synapse is experimentally robust and amenable to sophisticated genetic, imaging, electrophysiological, and pharmacological approaches. We detail the experimental design, electrophysiological recording procedure, and quantitative analysis necessary to determine the readily releasable pool size. This technique requires the use of a two-electrode voltageclamp recording configuration in elevated external $\mathrm{Ca}^{2+}$ with high frequency stimulation. We have used this assay to measure the readily releasable pool size and reveal that a form of homeostatic plasticity modulates this pool with synapse-specific and compartmentalized precision. This powerful approach can be utilized to illuminate the dynamics of synaptic vesicle trafficking and plasticity and determine how synaptic function adapts and deteriorates during states of altered development, stress and neuromuscular disease.
\end{abstract}

Keywords: Readily releasable pool, Neuromuscular junction, Synaptic vesicle pool, Two-electrode voltage clamp, Drosophila

[Background] The small fraction of synaptic vesicles (SVs) docked at the plasma membrane and primed for immediate fusion upon synaptic stimulation are referred to as the readily releasable vesicle pool (RRP), a key biophysical parameter that is a major determinant of synaptic strength. A reliable estimate of RRP is crucial for understanding the unique release properties within and across distinct types of synapses (Stevens, 2003; Abott and Regehr, 2004) and for distinguishing between different modes of short-term plasticity (Regehr, 2012). Estimation of the RRP has provided important insights into the dynamics of synaptic transmission and various forms of plasticity such as short-term depression (Hallermann et al., 2010; Regehr, 2012). At the Drosophila neuromuscular junction (NMJ), a powerful model of homeostatic plasticity has been established at this model glutamatergic synapse (Frank, 2013; 
Davis and Muller, 2015). An adaptive enhancement in presynaptic vesicle release is observed in response to reduced postsynaptic glutamate receptor functionality, a process referred to as Presynaptic Homeostatic Potentiation (PHP, Figure 1; Petersen et al., 1997; Frank et al., 2006; Davis and Muller, 2015). This increase in the size of the RRP is an important expression mechanism underlying PHP, and demonstrates that modulation of the RRP is a target of plasticity at synapses (Weyhersmuller et al., 2011; Muller et al., 2012; Chen et al., 2017; Goel et al., 2017; Kiragasi et al., 2017; Goel and Dickman, 2018; Li et al., 2018a and 2018b). In contrast, an inverse mode of homeostatic plasticity, referred to as Presynaptic Homeostatic Depression (Daniels et al., 2004), reduces presynaptic neurotransmitter release without any modulation to the size of the RRP (Gavino et al., 2015; Li et al., 2018b). A decrease in the RRP is observed in mutants that diminish presynaptic calcium influx (Muller et al., 2012; Muller et al., 2015; Wang et al., 2016). Alterations in other synaptic vesicle pools and recycling dynamics have been reported in mutations of synaptic genes in Drosophila, including shibire (dynamin), endophilin, synaptojanin, and synaptotagmin (Li and Schwarz, 1999; Delgado et al., 2000; Verstreken et al., 2002; Dickman et al., 2005; Bacaj et al., 2015). Thus, the RRP is an important parameter that establishes synaptic strength and can be selectively and dynamically modulated during plasticity.
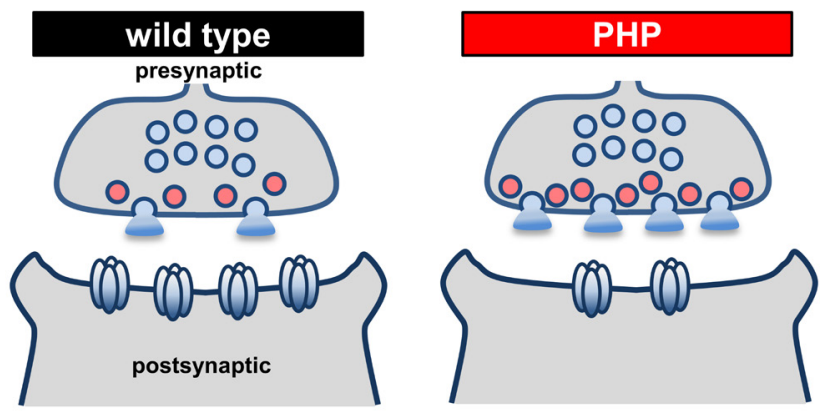

Figure 1. The RRP is enhanced during presynaptic homeostatic plasticity at the Drosophila

NMJ. Schematic of the fly NMJ at baseline (wild type) and following the expression of presynaptic homeostatic potentiation (PHP). Note that in response to loss of postsynaptic glutamate receptors, presynaptic neurotransmitter release is enhanced through increased RRP size (red vesicles). RRP size is quantified by the method detailed in this protocol.

A variety of anatomical and physiological methods for determining the size of various synaptic vesicle pool have been utilized, all loosely described as the RRP (Neher, 2015; Kaeser and Regehr, 2017). Each of these approaches have certain strengths and weaknesses. Anatomical measurements of the number of docked vesicles, defined as synaptic vesicles in close proximity to the active zone, have been assessed using electron microscopy and suggested to participate in the RRP (Schikorski and Stevens, 2001; Rizzoli and Betz, 2004). Though straightforward to measure, the main weakness of this method is that not all docked vesicles are primed and may not even be releasable. Therefore, not all docked vesicles contribute to the RRP physiologically, while the RRP might be capable of recruiting undocked vesicles (Kaeser and Regehr, 2017). A second approach for determining RRP size utilizes electrophysiology, where a hypertonic sucrose solution is applied near presynaptic terminals, inducing 
synaptic vesicle fusion. In this approach, osmotic shock is assumed to drive membrane fusion of vesicles that are located close to plasma membranes, but this measure assumes that osmotic shock and action potential-dependent calcium influx target the same vesicle pool. A third quantitative methodological approach is to induce a large and sustained elevation of presynaptic calcium by either chemical uncaging or through depolarization of the presynaptic terminal (Schneggenburger et al., 2002; Rizzoli and Betz, 2005). Finally, a fourth commonly used approach to estimate RRP size is to use a highfrequency train of action potentials to stimulate the synapse, which results in immediate fusion of readily releasable vesicles followed by rapid replenishment by endocytosis. It is assumed that the depression of the excitatory postsynaptic current (EPSC) amplitude is primarily caused by the depletion of the readily releasable quanta, after which a steady-state is established in which exocytosis is balanced by endocytosis. Thus, the RRP size can be estimated by calculating the cumulative EPSC amplitude (Figure 5; Schneggenburger et al., 1999). At the Drosophila NMJ, RRP size has been assayed either by using a stimulus train or by EPSC amplitude fluctuation analysis during single stimuli (Weyhersmuller et al., 2011; Muller et al., 2012; Penney et al., 2016; Wentzel et al., 2018). In this analysis, it is assumed that no contributions from the synaptic vesicle reserve pool (RP) contribute to the release dynamics during the initial rapid stimulation, and that the RP therefore is mobilized only after RRP depletion following strong stimulation intensity (Schneggenburger et al., 1999; Delgado et al., 2000; Denker and Rizzoli, 2010; Kaeser and Regehr, 2017). Indeed, the definition and make up of the RRP, RP and other vesicle pools ("exo-endo recycling", "docked and primed", etc.) vary depending on the synapse type, experimental approach, and experimental conditions used, leading to difficulty in making direct comparisons between the various vesicle pools (Rizzoli and Betz, 2005; Alabi and Tsein, 2012; Kaeser and Regehr, 2017). It is therefore important to be aware of the strengths and limitations in analyzing and interpreting differences between the various vesicle pools measured.

Here, we describe a detailed protocol to estimate the physiologically relevant RRP at the Drosophila NMJ. This method is based on TEVC recordings of a nerve-evoked train of EPSCs from muscle fibers of third-instar larvae. In particular, NMJs are stimulated at $60 \mathrm{~Hz}$ frequencies in $3 \mathrm{mM}$ external $\mathrm{Ca}^{2+}$ saline, followed by back-extrapolation of the cumulative EPSC to obtain an estimate of the RRP size. We have recently used this method to demonstrate that PHP signaling is compartmentalized, where RRP size can be specifically increased at only a subset of synapses within a single motor neuron (Li et al., 2018a). In addition, we successfully applied this technique to confirm that although RRP size is increased during PHP, RRP is unchanged in an inverse form of plasticity, PHD (Gavino et al., 2015; Li et al., 2018b). Thus, this method is a powerful approach that can be easily adapted to investigate SV dynamics in baseline synaptic transmission and plasticity, and to study how these properties may be altered in neuromuscular and motor neuron disease states using the powerful Drosophila NMJ as a model. 


\section{Materials and Reagents}

1. Suction (World Precision Instruments, catalog number: TW120-4) and recording (World Precision Instruments, catalog number: TW120F-4) micropipettes

2. $60 \mathrm{~mm}$ Petri dish

3. Glue gun (Tacklife, catalog number: GGO20AC)

4. Silver wire (Molecular Devices, Axon Accessories, catalog number: 1-HLA-005)

5. $10 \mathrm{ml}$ syringe (Thermo Fisher Scientific, Thermo Scientific ${ }^{\mathrm{TM}}$, catalog number: $\mathrm{S} 7510-10$ ) to fill micro-electrodes and apply negative pressure for suction electrode

6. MicroFil (World Precision Instruments, catalog number: MF28G67-5) to fill micro-electrodes

7. Drosophila third-instar larvae

8. $\mathrm{KCl}$ (Millipore Sigma, catalog number: P9333)

9. $\mathrm{NaCl}$ (Millipore Sigma, catalog number: S7653)

10. $\mathrm{MgCl}_{2} \cdot 6 \mathrm{H}_{2} \mathrm{O}$ (Millipore Sigma, catalog number: M2670)

11. $\mathrm{NaHCO}_{3}$ (Millipore Sigma, catalog number: $\mathrm{M} 2670$ )

12. Sucrose (Fisher Scientific, catalog number: S233-500)

13. D-(+)- Trehalose dihydrate (Millipore Sigma, catalog number: T9531)

14. HEPES (Millipore Sigma, catalog number: H3375)

15. $\mathrm{CaCl}_{2} \cdot 2 \mathrm{H}_{2} \mathrm{O}$ (Millipore Sigma, catalog number: C5080)

16. Regular liquid bleach (such as CloRox)

17. Modified hemolymph-like solution (HL-3) (see Recipes)

18. Recording electrode solution (see Recipes)

\section{Equipment}

1. PC Computer with a minimum requirement of $1.2 \mathrm{~GB}$ RAM, $2 \mathrm{GHz}$ processor, Windows 7,2 USB ports, $50 \mathrm{G}$ disk space

2. Upright microscope (such as Olympus, model: BX51W1) with $4 x$ air and 40x water dipping objectives, air table (TMC)

3. Micro-electrode puller (Sutter Instrument, model: P-97) with box platinum filament

Puller settings for Recording Electrode: Heat $=$ ramp + 10; Pull = 110; VEL. = 80; Time = 250;

Pressure $=500$

Setting for Suction Electrode: Heat $=$ Ramp $-15 ;$ Pull = 10; VEL. = 10; Time $=200 ;$ Pressure $=$ 500

4. ISO-Flex stimulator isolator unit (A.M.P.I.)

5. Micromanipulators

We use Siskiyou, including one manual manipulator for the suction electrode (Siskiyou, model: MX160L) and two motorized micromanipulators for TEVC (current injection and voltage; Siskiyou, models: MX7600 and MC1000C controller) 
6. Electrode headstages: Axon CNS; 0.1x and 10x (HS-9Ax0.1, HS-13Sx10)

7. Amplifier: Axoclamp 900A (Axon CNS, model: Axoclamp ${ }^{\mathrm{TM}}$ 900A)

8. Digidata board: Digidata 1440 A (Axon CNS)

9. Dissection tools and equipment:

a. 7x-45x Stereo microscope for dissection (AmScope, models: SM 1-B), LED light source (SciOptic, 3W*2PCs)

b. Drosophila larval dissection and recording chamber: 3" x 4" glass cover slip, magnetic tape, magnetic pins (Sullivan et al., 2000)

c. Scissors (Fine Science Tools, catalog number: 15000-08) and Forceps (Fine Science Tools, catalog number: 11295-20)

d. Razor (Darice, catalog number: B002C12EHM)

10. Microforge (Narishige, model: MF-830) for polishing suction electrode tip

11. Ice and ice bucket for maintaining ice cold modified HL-3 saline

\section{Software}

1. Acquisition

a. Clampex 10.7 software (Molecular Devices, Sunnyvale, CA, USA, licenses needed)

b. Axoclamp (Axon CNS, licenses needed)

2. Analysis

a. Clampfit (Molecular Devices, Sunnyvale, CA, USA: http://moleculardevices.app.box.com)

b. MiniAnalysis (Synaptosoft: www.synaptosoft.com/MiniAnalysis/)

c. SigmaPlot (licenses needed)

d. Microsoft Excel (licenses needed)

e. GraphPad Prism (licenses needed)

Note: MATLAB (MathWorks, USA) and other software can be used instead of $2 c, 2 d$ and $2 e$.

\section{Procedure}

A. Drosophila larval dissection and preparation

1. The Drosophila third-instar larval dissection has been described and video recorded (Brent, 2009), and the basic background for TEVC electrophysiological preparation, configuration, and recording has also been outlined (Kyriacou, 2010). Suction pipettes should be pulled with an opening of $\sim 5 \mu \mathrm{m}$, while the recording electrodes should be pulled with resistances of $\sim 15 \mathrm{M} \Omega$ (Figure 2). Polish the tip of the suction pipette using the microforge listed in the equipment, and fill both recording micropipettes with $3 \mathrm{mM} \mathrm{KCl}$ and the suction pipette with $3 \mathrm{mM} \mathrm{Ca}^{2+}$ modified HL-3 saline with microfill and syringe (Stewart et al., 1994; Dickman et al., 2005). Additional details of electrode preparation have been previously detailed in Zhang and Stewart (2010b). 




Figure 2. Examples of recording and suction electrodes. A. Representative recording electrode tip pulled by using the puller program described in Equipment. B. Representative suction electrode tip pulled by the program detailed in Equipment and polished to a diameter of $\sim 5 \mu \mathrm{m}$.

2. A magnetic recording chamber (shown in Figures $3 A$ and $3 B$; detailed in Ramachandran and Budnik, 2010) is used. The magnetic tape is necessary to attract the pins to the chamber and to hold down the filleted larvae while performing electrophysiology. While any glass can be used to form the foundation, a 3"x 4" chamber is a standard size that can be purchased cheaply and thus is what most experimentalist will prefer to use. A simple razor is used to manually cut out the diamond shape area of the magnetic tape in the middle of the chamber, and a simple glue gun is used to seal the edges between the glass and the magnetic tape around the diamond cut out. This glue seal is used to hold the saline around the dissected larvae while performing electrophysiology. Alternatively, a $60 \mathrm{~mm}$ dish lined with Sylgard and sharp pins can be used to impale the filleted larvae for electrophysiological preparation (Singh and Wu, 1999; Budnik and Ruiz-Canada, 2006; Imlach and McCabe, 2009; Zhang and Stewart, 2010a and 2010c; Itoh et al., 2016; Bykhovskaia and Vasin, 2017). However, we recommend and prefer the magnetic chamber because of the gentler dissection, the lower saline volume necessary and the superior microelectrode accessibility.

3. Dissect third-instar larvae in ice-cold $\mathrm{Ca}^{2+}$ free modified $\mathrm{HL}-3$ saline (to prevent muscle movement during dissection). The recipe of the $\mathrm{Ca}^{2+}$ free modified $\mathrm{HL}-3$ saline is the same as the modified $\mathrm{HL}-3$ without $\mathrm{CaCl}_{2}$. Loosely pin the larvae (Figures $3 \mathrm{~A}$ and $3 \mathrm{~B}$ ), remove the guts, trachea and ventral nerve cord from the larval body walls. Cut all motor nerves that originated from the ventral nerve cord (Figure 3C) and perfuse the preparation several times with fresh HL-3 saline via pipetting. Ensure that the motor nerves are not cut too short, as this may result in difficulty with suctioning and stimulating the nerve. Finally, fill the chamber with $3 \mathrm{mM} \mathrm{Ca}^{2+}$ modified HL-3 saline.

4. Orient the NMJ preparation on the microscope stage for optimal electrode positioning (Figures $3 \mathrm{C}$ and $4 \mathrm{~A})$. 


\section{bĭ̈-protocol}

A
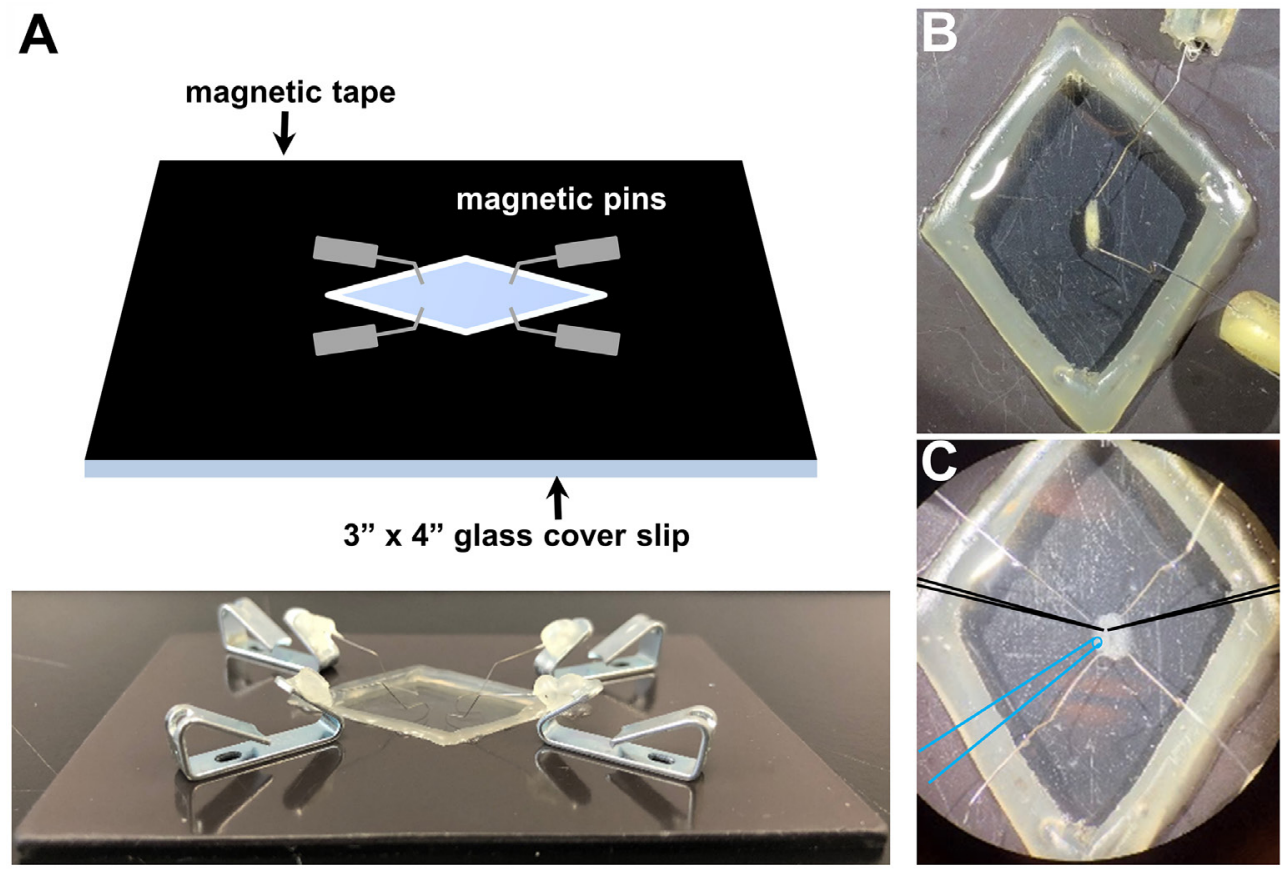

Figure 3. Drosophila larvae before and after dissection in the recording chamber. A. Representative schematic (top) and picture (bottom) of the recording chamber used for larval dissection and electrophysiology with magnetic pins. Representative images of a third-instar larvae before (B) and after dissection (C) using this system. Note that optimal positioning of recording electrodes (schematized in black) and the stimulation pipette (schematized in blue).

\section{B. Electrophysiological recordings}

1. Place the larval preparation and the tips of the three electrodes in the middle field of view using the $4 x$ objective, and lower the electrodes closer to the plane of the preparation. We typically record from muscle 6 at abdominal segments $A 2$ or A3. Switch to the 40x objective, move the target muscle close to the electrodes, and lower the suction electrode near the nerve bundle. Provide a small negative pressure through the syringe to withdraw the nerve into the pipette. Use suction to establish a seal between the suction electrode and the motor nerve that innervates the muscle segment (Figure 4B). 
A

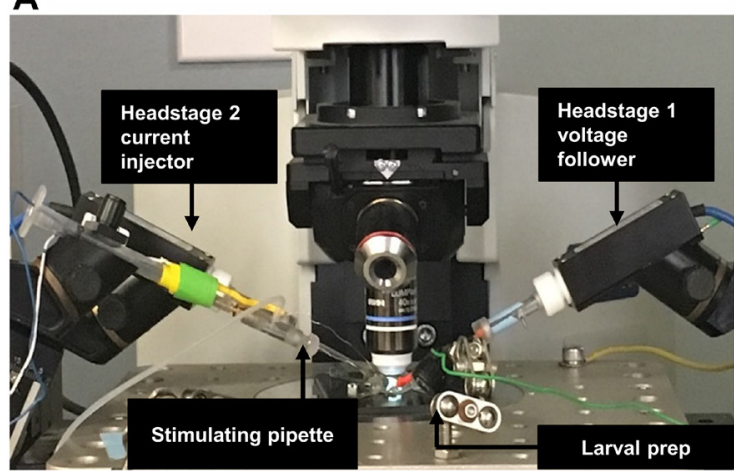

B

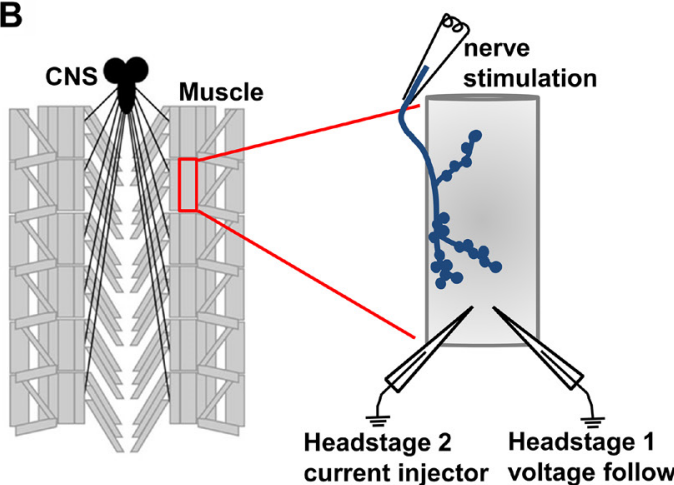

Figure 4. Schematic illustrating the TEVC recording configuration of the third-instar larval NMJ. A. Picture of the electrophysiology setup, with electrodes placed to stimulate and record from the larval preparation. B. Schematic of a dissected third-instar larvae with a detailed view of the muscle $6 \mathrm{NMJ}$ after all electrodes are properly positioned. A stimulating electrode is used to suck the severed motor nerve innervating the muscle segment to be recorded from (top) and the recording electrodes are used for current injection or voltage following (bottom). The presynaptic nerve terminal (blue) is shown in a classical "beads on a string" bouton structure.

2. Move the recording electrodes right above muscle 6 , which is innervated by the motor nerve isolated in the stimulation electrode (Figures $3 \mathrm{C}$ and $4 \mathrm{~B}$ ). Set electrode baseline potentials to 0 by resetting the bridge balance and pipette offset. Chloride the silver wire in the recording pipette if the pipette offset value is higher than $100 \mathrm{mV}$.

3. Digitize electrophysiological sweeps at $10 \mathrm{kHz}$ by setting the sampling rate in the Clampex software, and filter the lowpass signal at $1 \mathrm{kHz}$.

4. Impale the muscle 6 fiber with the voltage follower electrode (HS-9Ax0.1) and record the resting potential ( $V_{\text {rest }}$ ). Inject a $500 \mathrm{msec}$ pulse of $-1 \mathrm{nA}$ current to measure the muscle input resistance $\left(R_{\text {in }}\right)$ by Ohm's law: $R_{\text {in }}=\frac{V_{\text {rest }}}{-1 n A}$. Reject recordings with $V_{\text {rest }}$ more depolarized than $-60 \mathrm{mV}$ or more hyperpolarized than $-85 \mathrm{mV}$, or if the $R_{\text {in }}$ is less than $5 \mathrm{M} \Omega$.

5. Impale the same muscle fiber with the current injection electrode (HS-13Sx10) and wait for 15$20 \mathrm{~s}$ until the voltage potential readings from both electrodes become stable (both more hyperpolarized than $-55 \mathrm{mV}$ ). Switch to TEVC mode, clamp the muscle membrane potential at $-70 \mathrm{mV}$, and monitor the leak current. Reject recordings with leak current values higher than 10 nA. Miniature excitatory postsynaptic currents (mEPSCs) are recorded in the absence of any stimulation for one minute with a typical gain value of 80 . Stimulate motor axons to elicit excitatory postsynaptic currents (EPSCs) using an ISO-Flex stimulus isolator (A.M.P.I.) to modulate the amplitude of stimulatory currents. Adjust intensity for each recording to consistently elicit responses from both neurons innervating the muscle segment, but avoid overstimulation, in which case responses with multiple peaks can be initiated.

6. Switch to the protocol necessary to measure the RRP using the same optimal stimulation intensity established in the previous step. The RRP protocol stimulates evoked responses and 
records EPSCs using a $60 \mathrm{~Hz}$ train of 30 stimuli in $3 \mathrm{mM}$ extracellular $\mathrm{Ca}^{2+} \mathrm{HL}-3$ (Figure 5). The train sweeps were repeated for five times in each recording. The train of stimulus at a high $\mathrm{Ca}^{2+}$ concentration leads to a progressive depression of EPSC amplitude during the stimulation paradigm due to a depletion of readily releasable vesicles (Figure 5B).

A

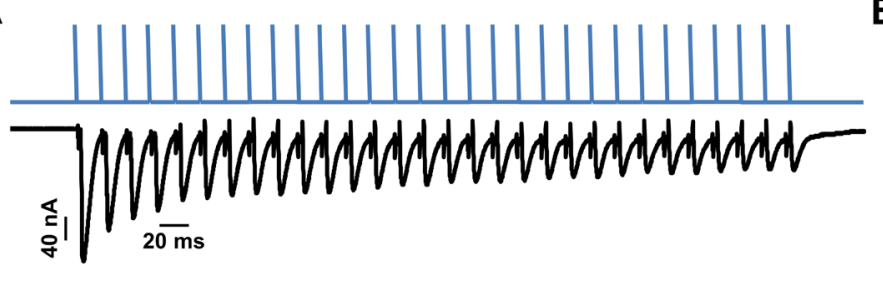

B

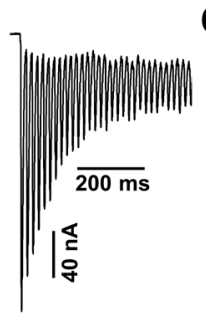

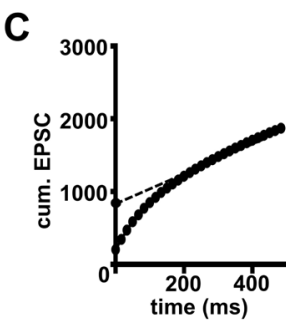

Figure 5. Stimulation and analysis paradigm for estimating the RRP. A. Representative stimulation paradigm (above) and electrophysiological responses (below) of the $60 \mathrm{~Hz} / 30$ stimuli protocol necessary to determine the RRP. B. Representative EPSC recording during a $60 \mathrm{~Hz}$ stimulus train at a typical NMJ. C. Average cumulative EPSC amplitude plotted as a function of time. A line fit to the 18-30th stimuli was back extrapolated to time 0 ; this $y$-intercept value is divided by the mEPSC value to determine the size of the RRP.

\section{$\underline{\text { Data analysis }}$}

1. Amplify and digitize the recorded signal using an Axoclamp $900 \mathrm{~A}$ amplifier in combination with the Digidata 1440A acquisition system and pClamp 10.5 software. Appropriate software and hardware from other vendors can also be used.

2. Analyze the amplitude of mEPSC events using Mini Analysis (Synaptosoft, Figure 6; additional details of using this program can be found here: http://www.synaptosoft.com/MiniAnalysis/Tutorial/index files/frame.htm). Briefly, open the ".abf" file in Mini Analysis and increase Gain to 200 (Figure 6) to clearly visualize miniature EPSC events. Use the "Analysis" setting shown in Figure 6 to accurately capture and quantify mini events. Export the results of $\sim 100$ events to Microsoft Excel and average the mEPSC amplitude of at least 100 events from each recording to get the average mEPSC amplitude for that particular muscle cell.

3. Use SigmaPlot and Clampfit to analyze the amplitude of each EPSC in the stimulus train.

a. Transfer the sweeps of train stimulation from each cell to data points in Clampfit.

b. Calculate EPSC amplitudes by taking the difference between peak and baseline before stimulus onset of a given EPSC manually (using Mini-Analysis) or using an optimized program (user defined code) in SigmaPlot or MATLAB. Such a custom-written script can be provided upon request.

c. Obtain the average amplitude of the 30 EPSCs from five sweeps using SigmaPlot. Then tabulate $19^{\text {th }}-30^{\text {th }}$ twelve averaged EPSC amplitudes paired with the corresponding time 
point onto a new result sheet in Clampfit.

4. We are estimating the RRP size by using the cumulative EPSC amplitude method as first described by Schneggenburger et al., 1999. This method was performed at the Calyx of Held in the mammalian central nervous system. Briefly, a train of high frequency stimulation in saline containing elevated calcium concentration introduces a fast depression in synaptic responses, indicative of RRP depletion. A relatively steady state phase follows this initial rapid depression, where endocytosis and exocytosis rates are assumed to be balanced due to vesicle recycling and the contribution of vesicles mobilized from other pools. This has been adapted and applied to the Drosophila NMJ (Hallermann et al., 2010; Weyhersmuller et al., 2011; Muller et al., 2012; Davis and Muller, 2015; Muller et al., 2015; Chen et al., 2017; Kiragasi et al., 2017; Li et al., 2018a and 2018b).

a. Calculate the cumulative EPSC from a line fit to the linear phase (stimuli \#18-30) of the EPSC data back-extrapolated to time 0 (Figure 5).

b. Estimate RRP size by normalizing the ratio of the cumulative EPSC value at time 0 and the average mEPSC amplitude in Microsoft Excel. Finally, copy the mEPSC, cumulative EPSC and RRP size values to GraphPad Prism and perform appropriate statistical analyses to assess significance ( $\mathrm{Li}$ et al., 2018a and 2018b; Materials and Methods). Generally, compare multiple data sets with a one-way ANOVA followed by a Tukey's multiple comparison test. A good illustration of this analysis can be found in Figures $5 \mathrm{~B}$ and $5 \mathrm{C}$ of $\mathrm{Li}$ et al. (2018a). Optimal recordings of 6-10 NMJs from at least three different larvae of the same genotype are typically used for final analysis.


Figure 6. Data analysis to determine mEPSC amplitude necessary to estimate RRP size.

Screenshot of the Mini-Analysis Program showing a representative analysis of mEPSC amplitude from acquired electrophysiological traces. Shown at right are the Mini Analysis settings used. 


\section{$\underline{\text { Notes }}$}

A cautionary point is in order regarding the acquisition of mEPSCs and EPSCs. It can be difficult to accurately record mEPSCs and EPSCs from the same muscle cell. This is due to the necessity for low noise to resolve small mEPSC events in the TEVC configuration, which requires two $0.1 \mathrm{x}$ headstages. While this is optimal for resolving small mEPSC events, these low gain headstages are unable to voltage clamp excitability of the muscle during the large currents driven by evoked stimulation at elevated extracellular $\mathrm{Ca}^{2+}$ recording conditions $(3 \mathrm{mM})$. In these conditions, we use a $0.1 \mathrm{x}$ headstage for voltage follower and a $10 \mathrm{x}$ headstage for current injection to provide sufficient current to clamp the muscle. The installment noise with the $10 \mathrm{x}$ current injection headstage is suboptimal for resolving mEPSC signals, particularly the small miniature events induced by genetic or pharmacological perturbations to the postsynaptic glutamate receptors. Hence, the average mEPSC amplitudes are typically obtained from the same genotype but averaged between different larvae and NMJs.

\section{$\underline{\text { Recipes }}$}

1. Modified hemolymph-like solution (HL-3); $\mathrm{pH} 7.2$

\begin{tabular}{|l|l|}
\hline Chemical & Final concentration (mM) \\
\hline $\mathrm{NaCl}$ & 70 \\
\hline $\mathrm{KCl}$ & 5 \\
\hline $\mathrm{MgCl}_{2}$ & 10 \\
\hline $\mathrm{NaHCO}_{3}$ & 10 \\
\hline Sucrose & 115 \\
\hline Trehalose & 5 \\
\hline $\mathrm{HEPES}$ & 5 \\
\hline $\mathrm{CaCl}_{2}$ & 3 \\
\hline
\end{tabular}

Note: Prepared solution without calcium can be stored at $-20{ }^{\circ} \mathrm{C}$ and thawed before use. $\mathrm{CaCl}_{2}$ is added to the saline right before beginning the experiments. The dissolved HL-3 containing the desired $\mathrm{Ca}^{2+}$ concentration can be stored at $4{ }^{\circ} \mathrm{C}$ and reused for up to 3-4 days.

2. Recording electrode solution

\begin{tabular}{|l|l|}
\hline Chemical & Final concentration \\
\hline $\mathrm{KCl}$ & $3 \mathrm{M}$ \\
\hline
\end{tabular}




\section{Acknowledgments}

Work in the Dickman lab is funded by a grant from the National Institutes of Health (NS091546). We thank Martin Müller (University of Zürich) for insightful discussions and technical insights into TEVC recording and RRP analysis.

\section{Competing interests}

The authors declare no conflicts or other competing interests.

\section{$\underline{\text { References }}$}

1. Abott, L. F. and Regehr, W. G. (2004). Synaptic computation. Nature 431(7010): 796-803.

2. Alabi, A. A. and Tsein, R. W. (2012). Synaptic vesicle pools and dynamics. Cold Spring Harb Perspect Biol 4: a013680.

3. Bacaj, T., Wu, D., Burre, J., Malenka, R. C., Liu, X. and Sudhof, T. C. P. B. (2015). Synaptotagmin-1 and -7 are redundantly essential for maintaining the capacity of the readilyreleasable pool of synaptic vesicles. PloS Biol 13: e1002267.

4. Brent, J. R., Werner, K. M. and McCabe, B. D. (2009). Drosophila larval NMJ dissection. J Vis Exp 24(e1107).

5. Budnik, V. and Ruiz-Canada, C. (2006). The fly neuromuscular junction: structure and function. 75.

6. Bykhovskaia, M. and Vasin, A. (2017). Electrophysiological analysis of synaptic transmission in Drosophila. Wiley Interdiscip Rev Dev Biol 6(5).

7. Chen, X., Ma, W., Zhang, S., Paluch, J., Guo, W. and Dickman, D. K. (2017). The BLOC-1 subunit pallidin facilitates activity-dependent synaptic vesicle recycling. eNeuro 4(1): 1-18.

8. Daniels, R. W., Collins, C. A., Gelfand, M. V., Dant, J., Brooks, E. S., Krantz, D. E. and DiAntonio, A. (2004). Increased expression of the Drosophila vesicular glutamate transporter leads to excess glutamate release and a compensatory decrease in quantal content. $J$ Neurosci 24(46): 10466-10474.

9. Davis, G. W. and Muller, M. (2015). Homeostatic control of presynaptic neurotransmitter release. Ann Rev Physiol 77: 251-270.

10. Delgado, R., Maureira, C., Oliva, C., Kidokoro, Y. and Labarca, P. (2000). Size of vesicle pools, rates of mobilization, and recycling at neuromuscular synapses of a Drosophila mutant, shibire. Neuron 28(3): 941-953.

11. Denker, A. and Rizzoli, S. O. (2010). Synaptic vesicle pools: an update. Front Synaptic Neurosci 2: 135. 
12. Dickman, D. K., Horne, J. A., Meinertzhagen, I. A. and Schwarz, T. L. (2005). A slowed classical pathway rather than kiss-and-run mediates endocytosis at synapses lacking synaptojanin and endophilin. Cell 123(3): 521-533.

13. Frank, C. A. (2013). Homeostatic plasticity at the Drosophila neuromuscular junction. Neuropharmacology 78: 63-74.

14. Frank, C. A., Kennedy, M. J., Goold, C. P., Marek, K. W. and Davis, G. W. (2006). Mechanisms underlying the rapid induction and sustained expression of synaptic homeostasis. Neuron 52(4): 663-677.

15. Gavino, M. A., Ford, K. J., Archila, S. and Davis, G. W. (2015). Homeostatic synaptic depression is achieved through a regulated decrease in presynaptic calcium channel abundance. eLife 4: e05473.

16. Goel, P. and Dickman, D. (2018). Distinct homeostatic modulations stabilize reduced postsynaptic receptivity in response to presynaptic DLK signaling. Nat Commun 9(1): 1856.

17. Goel, P., Li, X. and Dickman, D. (2017). Disparate postsynaptic induction mechanisms ultimately converge to drive the retrograde enhancement of presynaptic efficacy. Cell Rep 21(9): 23392347.

18. Hallermann, S., Heckmann, M. and Kittel, R. J. (2010). Mechanisms of short-term plasticity at neuromuscular active zones of Drosophila. HFSP Journal 4: 72-84.

19. Imlach, W. and McCabe, B. D. (2009). Electrophysiological methods for recording synaptic potentials from the NMJ of Drosophila larvae. J Vis Exp 24: e1109.

20. Itoh, K., Komatsu, A. and Nishihara, S. (2016). Electrophysiological recording in the Drosophila larval muscle.

21. Kaeser, P. S. and Regehr, W. G. (2017). The readily releasable pool of synaptic vesicles. Curr Opin Neurobiol 43: 63-70.

22. Kiragasi, B., Wondolowski, J., Li, Y. and Dickman, D. K. (2017). A presynaptic glutamate receptor subunit confers robustness to neurotransmission and homeostatic potentiation. Cell Rep 19(13): 2694-2706.

23. Kyriacou, C. (2010). Drosophila neurobiology: a laboratory manual. Cold Spring Harbor Laboratory Press.

24. Li, J. and Schwarz, T. L. (1999). Genetic evidence for an equilibrium between docked and undocked vesicles. Philos Trans R Soc Lond B Biol Sci 354(1381): 299-306.

25. Li, X., Goel, P., Chen, C., Angajala, V., Chen, X. and Dickman, D. (2018a). Synapse-specific and compartmentalized expression of presynaptic homeostatic potentiation. eLife 7: e34338.

26. Li, X., Goel, P., Wondolowski, J., Paluch, J. and Dickman, D. (2018b). A glutamate homeostat controls the presynaptic inhibition of neurotransmitter release. Cell Rep 23(6): 1716-1727.

27. Muller, M., Genc, O. and Davis, G. W. (2015). RIM-binding protein links synaptic homeostasis to the stabilization and replenishment of high release probability vesicles. Neuron 85(5): $1056-$ 1069. 
28. Muller, M., Liu, K. S., Sigrist, S. J. and Davis, G. W. (2012). RIM controls homeostatic plasticity through modulation of the readily-releasable vesicle pool. J Neurosci 32(47): 16574-16585.

29. Neher, E. (2015). Merits and limitations of vesicle pool models in view of heterogeneous populations of synaptic vesicles. Neuron 87: 1131-1142.

30. Penney, J., Tsurudome, K., Liao, E. H., Kauwe, G., Gray, L., Yanagiya, A., M, R. C., Sonenberg, N. and Haghighi, A. P. (2016). LRRK2 regulates retrograde synaptic compensation the Drosophila neuromuscular junction. Nat Commun 7: 12188.

31. Petersen, S. A., Fetter, R. D., Noordermeer, J. N., Goodman, C. S. and DiAntonio, A. (1997). Genetic analysis of glutamate receptors in Drosophila reveals a retrograde signal regulating presynaptic transmitter release. Neuron 19(6): 1237-1248.

32. Ramachandran, P. and Budnik, V. (2010). Dissection of Drosophila larval body-wall muscles. Cold Spring Harb Protoc 2010(8): pdb.prot5469.

33. Regehr, W. G. (2012). Short-term presynaptic plasticity. Cold Spring Harb Perspect Biol 4(7): a005702.

34. Rizzoli, S. O. and Betz, W. J. (2004). The structural organization of the readily releasable pool of synaptic vesicles. Science 303: 2037-2039.

35. Rizzoli, S. O. and Betz, W. J. (2005). Synaptic vesicle pools. Nat Rev Neurosci 6(1): 57-69.

36. Schikorski, T. and Stevens, C. F. (2001). Morphological correlates of functionally defined synaptic vesicle populations. Nat Neurosci 4: 391-395.

37. Schneggenburger, R., Meyer, A. C. and Neher, E. (1999). Released fraction and total size of a pool of immediately available transmitter quanta at a calyx synapse. Neuron 23: 399-409.

38. Schneggenburger, R., Sakaba, T. and Neher, E. (2002). Vesicle pools and short-term synaptic depression: lessons from a large synapse. Trends Neurosci 25: 206-212.

39. Singh, S. and Wu, C.-F. (1999). lonic currents in the larval muscles of Drosophila. Neuromuscular Junctions in Drosophila. Int Rev Neurobiol 43: 191-220.

40. Stevens, C. F. (2003). Neurotransmitter release ar central synapses. Neuron 40: 381-388.

41. Stewart, B. A., Atwood, H. L., Renger, J. J., Wang, J. and Wu, C. F. (1994). Improved stability of Drosophila larval neuromuscular preparations in haemolymph-like physiological solutions. $J$ Comp Physiol A 175(2): 179-191.

42. Sullivan, W., Ashburner, M. and Hawley, R. S. E. (2000). Drosophila protocols. Cold Spring Harbor Laboratory Press.

43. Verstreken, P., Kjaerulff, O., Lloyd, T. E., Atkinson, R., Zhou, Y., Meinertzhagen, I. A. and Bellen, H. J. (2002). Endophilin mutations block clathrin-mediated endocytosis but not neurotransmitter release. Cell 109(1): 101-112.

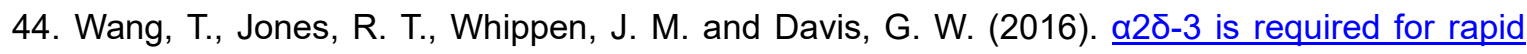
transsynaptic homeostatic signaling. Cell Rep 16(11): 2875-2888.

45. Wentzel, C., Delvendahl, I., Sydlik, S., Georgiev, O. and Muller, M. (2018). Dysbindin links presynaptic proteasome function to homeostatic recruitment of low release probability vesicles. Nat Commun 9(1): 267. 
46. Weyhersmuller, A., Hallermann, S., Wagner, N. and Eilers, J. (2011). Rapid active zone remodeling during synaptic plasticity. $J$ Neurosci 31(16): 6041-6052.

47. Zhang, B. and Stewart, B. (2010a). Electrophysiological recording from Drosophila larval bodywall muscles. Cold Spring Harb Protoc (9).

48. Zhang, B. and Stewart, B. (2010b). Fabrication of microelectrodes, suction electrodes, and focal electrodes. Cold Spring Harb Protoc (9).

49. Zhang, B. and Stewart, B. (2010c). Synaptic physiology at the Drosophila neuromuscular junction (chapter 12). Drosophila Neurobiology: A Laboratory Manual. 